\title{
Performance Analysis of Advanced Encryption Standard on FPGA
}

\author{
Lokesh Namdeo \\ Dept. of Electronics \& Communication Engineering \\ Sagar Institute of Research \& Technology \\ Bhopal (M. P.)
}

\author{
Himanshu Nautiyal \\ Dept. of Electronics \& Communication Engineering \\ Sagar Institute of Research \& Technology \\ Bhopal (M. P.)
}

\begin{abstract}
The Advanced Encryption Standard is the data security standard referred to as Federal Information Processing Standard 197 (FIPS 197) acquired worldwide by several private and public sectors for protective needs of data storage and secure data application from mobile consumer products to high end user. Most of the AES implementation for reconfigurable devices however based on the configurable logic such as flip-flops and lookup tables. In this paper, all the three modules i.e. AES - 128, AES - 192 and AES - 256 are implemented on Spartan 3 XC3S1000L speed grade -4 FPGA. All the three modules are implemented with four techniques namely, sequential 3 Stage, sequential 2 stage, pipelined and combinational and then a performance of the three techniques is evaluated. At last a combined AES supporting all the three AES types is also implemented using combinational technique. It is observed from the implementations that sequential 2 stage technique is most area efficient technique, whereas pipelined technique is most time efficient technique.
\end{abstract}

\section{Keywords}

AES, FPGA, Rijndael Algorithm, FIPS - 197, Pipelined architecture.

\section{INTRODUCTION}

The Advanced Encryption Standard denotes the Rijndael algorithm that can access 128 bits of data blocks using keys of 128, 192, 256-bit length. The Advanced Encryption Standard encipher exchanges data to an unintelligible form using the cipher key and the Advanced Encryption Standard decipher exchanges the cipher text back to plain text using similar cipher key. In Advanced Encryption Standard (AES) similar key is used for both decryption and encryption. Cryptography is an effective way of protecting sensitive information as it is stored on media or transmitted through network communication paths. Although the ultimate goal of cryptography, and the mechanism that make it up, is to hide information from unauthorized individuals. The approach of the project is towards advanced encryption standard algorithm that widely used algorithm in nowadays cryptographic technique. Advanced Encryption Standard decryption and encryption are concerned on four various transformations applied again and again in a specific input data consequences and the data flows of decryption and encryption are not similar. An expansion key module is used to distribute keys for several iterations of the AES algorithm. Depending on the input key length, the number of iterations of the Advanced Encryption Standard algorithm will vary.AES algorithm is an iterated symmetric block cipher which means that it works by repeating the same defined steps multiple times. In cryptography, AES is called as Rijndael Encryption algorithm that is capable of protecting sensitive information. [1-4]

\section{AES FRAME WORK}

Table 1 shows the structure of Rijndael Algorithm adopted by AES. AES uses the data block of 128 bits and Cipher key of 128,192 or 256 . The number of rounds for AES 128, AES 192 and AES 256 are 10, 12 and 14 respectively. In each round a same set of operations are performed [2-3].

Table 1: Structure of AES

\begin{tabular}{|c|c|c|c|}
\hline \multirow{2}{*}{ AES type } & \multicolumn{3}{|c|}{ Structure of AES } \\
\cline { 2 - 4 } & Cipher Key Length & Data Block Size & $\begin{array}{c}\text { Number of } \\
\text { rounds (N) }\end{array}$ \\
\hline AES 128 & 128 & 128 & 10 \\
\hline AES 192 & 192 & 128 & 12 \\
\hline AES 256 & 256 & 128 & 14 \\
\hline
\end{tabular}

\subsection{Encryption in AES}

The process of encryption begins with the conversion of 128bit data to a 4 x 4 state matrix of 16 bytes. Similarly, the input cipher key is also converted to a 4 x 4 matrix of 16 bytes. For AES - 128, the cipher key matrix size of 16 bytes is same the cipher key size 128 bits (16 bytes). For AES 192 and AES 256 , the first 128 bits (16 bytes) are used in first round and the remaining bits are used in next round. The set of operations performed in each round are listed below. $[7,8]$

1. Add_Round_key: in this operation, the state matrix is xored with the cipher key matrix and a new state matrix is formed.

2. Sub_bytes: in this operation, each byte of state matrix is replaced by a byte form a 256-byte table called SBOX.

3. Shift rows: State matrix has 4 rows, in this operation the first row is not shifted, the second row is shifted left cyclically by 1 byte, the third row is shifted left cyclically by 2 bytes and the fourth row is left shifted cyclically by 3 bytes.

Mix_column: A linear transformation is used in this process The mix column is process is used in 4 columns.

\section{IMPLEMENTATION OF AES}

In this work, we have implemented the three AES modes namely; AES 128, AES 196 and AES 256 on Spartan FPGA using four different techniques namely Sequential 3 Stage, sequential 2 stage, Pipelined and Combinational. All the four techniques have their own pros and cons which will be discussed in following sections. 


\subsection{Stage Sequential Implementation of AES}

Figure 1 depicts the sequential 3 - stage implementation of AES. In this technique first the plain text is converted into state array and then it is XORED with input cipher key (cipher key length is 128, 196 and 256 for AES 128, AES 192 and AES 256 respectively.) in the add_round process. Then the rounding process (round 1 to round $\mathrm{N}-1$ ) is implemented using a unit which has four sub units in it namely: Sub_Bytes, Shift_Rows, Mix_Column and Add_Round_Key. Then the last round is implemented using another unit which has only three sub units namely: Sub_Bytes, Shift_Rows and Add_Round_Key. This is a low area implementation as it uses the same hardware for round 1 to round $\mathrm{N}-1$. [10]

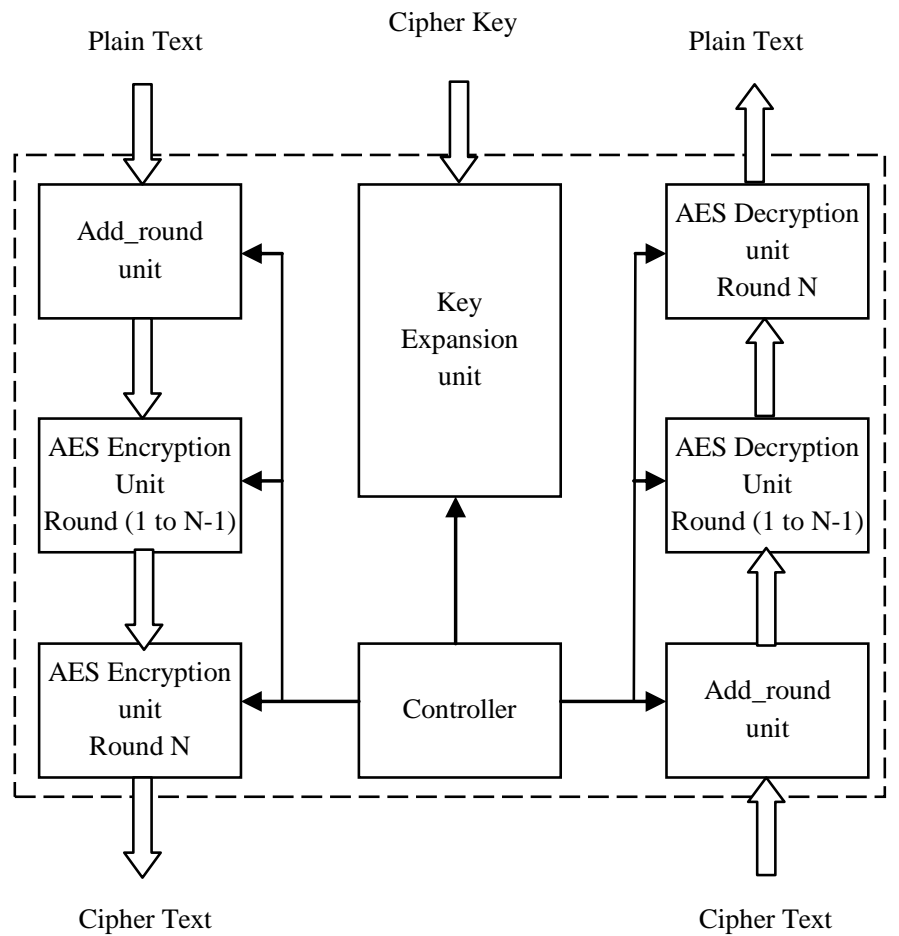

Figure 1: 3-Stage Sequential Implementation of AES

\subsection{Stage Sequential Implementation of}

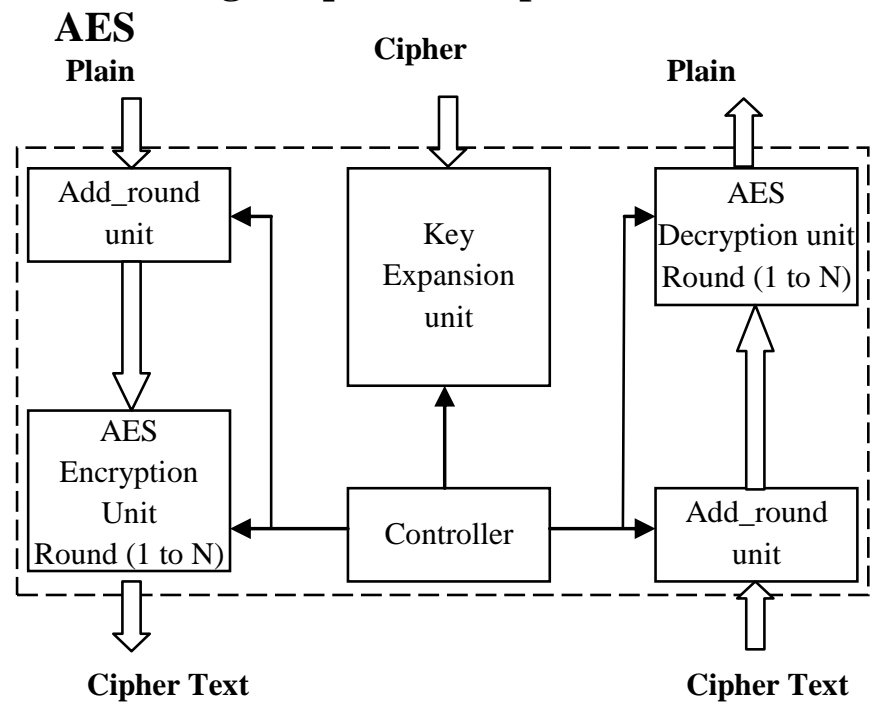

Figure 2: 2-Stage Sequential Implementation of AES
Figure 2 shows the implementation of 2 stage sequential machine for AES. In this first the plain text is converted to state matrix and then it is XORED with the input cipher key. Then from round 1 to round N-1 the state array goes from the four subunits namely: Sub_Bytes, Shift_Rows, Mix_Column and Add_Round_Key. In the last round ( ${ }^{\text {th }}$ round) the mix column process is not used and the state array goes through only three remaining subunits. This technique also uses hardware sharing as the four subunits are used $\mathrm{N}$ times to generate the final cipher text. In this technique, the resource usage will further reduce as compared to 3 stage sequential implementation as it does not use one more unit for the last round. [10]

\subsection{Combinational Implementation of AES}

Combinational implementation is a high-speed implementation technique, in this method all the rounds are implemented using separate rounding units, so a sum of $\mathrm{N}$ rounding units are required to implement the complete design.

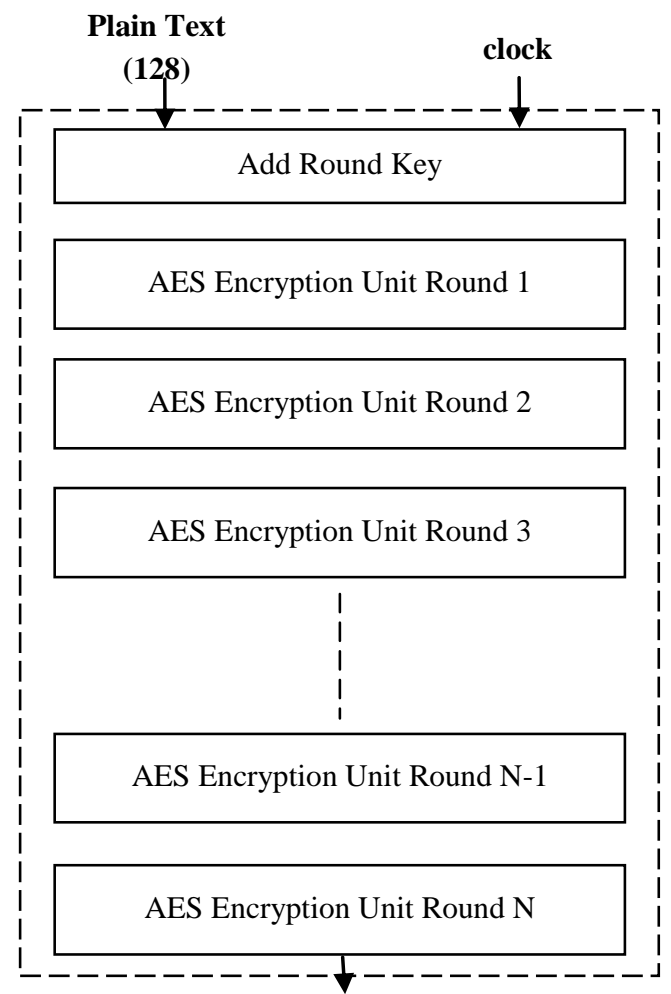

Cipher Text (128)

Figure 3: Combinational Implementation of AES

Figure 3 shows the hardware equivalent of combinational module of AES. This technique requires huge area (resources) as it used 10 rounding units compared to 2 rounding units in sequential 3 stage machine and single rounding unit in 2-stage sequential machine. Also, the area requirement grows with number of rounds, the number of rounds for AES 128, AES 192 and AES 256 are 10,12 and 14 respectively which means 10 rounding units are required to implement AES 128, 12 rounding unit are required to implement AES 192 and 14 rounding units are required to implement AES 256. The major advantage of combinational technique is low delay as $\mathrm{N}$ units are working in parallel compared to a single or two units.

Internal architecture of round 1 to round $\mathrm{N}-1$ is same and it uses four subunits namely: Sub_Bytes, Shift_Rows, 
Mix_Column and Add_Round_Key. Figure 4 shows the internal architecture of round 1 to round $\mathrm{N}-1$.

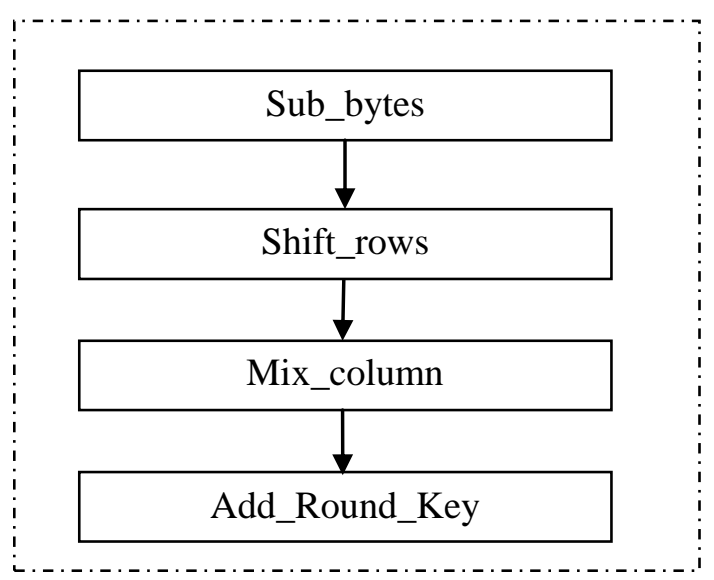

Figure 4: Round 1 to Round N-1 of AES combinational

Internal architecture for round $\mathrm{N}$ is different from round 1 to round $\mathrm{N}-1$ as it does uses mix_column subunit and uses only the remaining three subunits. Figure 5 shows the internal structure of round $\mathrm{N}$ of AES combinational.

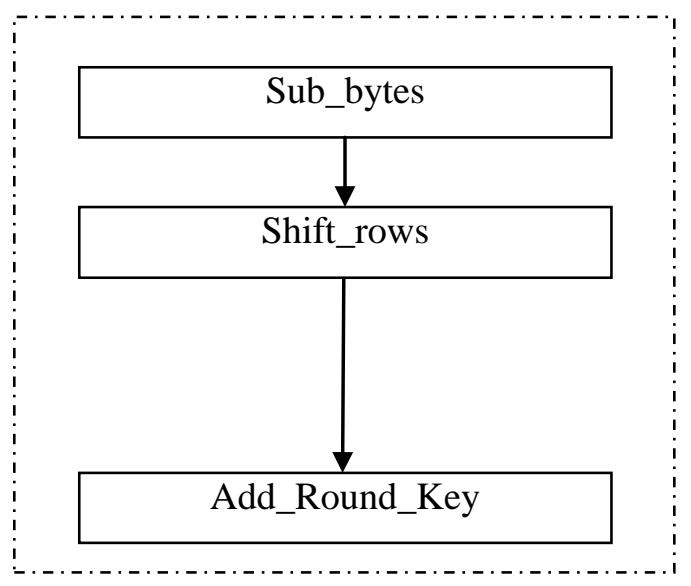

Figure 5: Round N of AES combinational

\subsection{Pipelined Implementation of AES}

The combinational technique is used to decrease the delay and increase throughput. Further increase in throughput can be obtained by using pipelining technique. In this technique, a 128-bit register is used after each rounding unit to store the data temporarily. Figure 6 shows the internal structure of pipelined architecture of AES.

At clock 1 the input data packet 1 is add_rounded and then stored in a 128-bit register. Then in the next clock the stored data in register 1 is assigned to round 1 unit and stored in register 2 and simultaneously the next data packet 2 is add_rounded and stored in register 1 in the same clock duration. This process continues for all the rounds. After $\mathrm{N}$ clock pulses the final cipher text 1 is obtained and then in the next clock cipher text packet 2 is obtained corresponding to the input packet 2 . Now the cipher text packets will continue to stream at every clock pulse. The maximum operating frequency is derived from the delay of one round rather than the delay of sum of the all the rounds as in combinational design. The major advantage of pipelined technique is high operating speed and frequency. The internal architecture of rounds is same as that of combinational design shown in figure 4 and 5 .

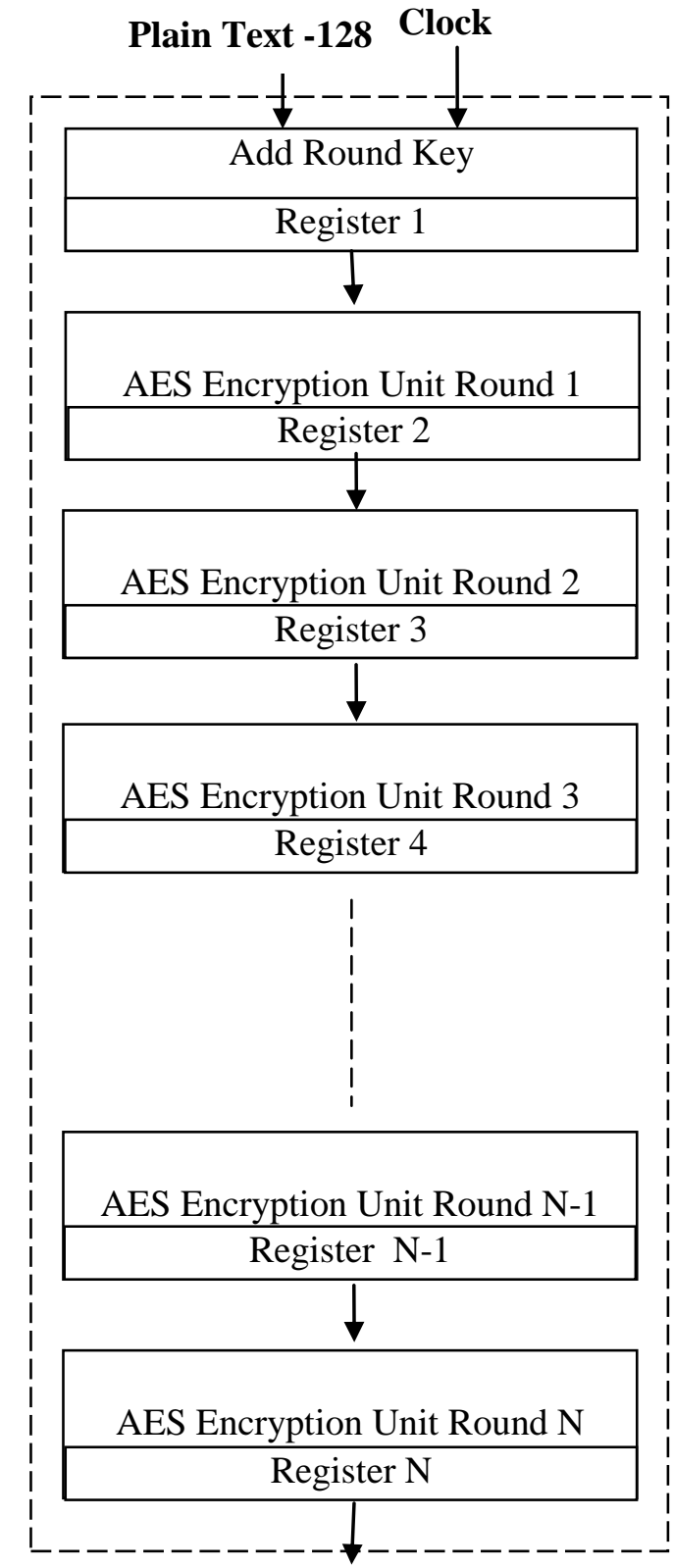

\section{Cipher Text (128)}

Figure 6: Round N of AES combinational

\section{RESULT}

In this section, various designs are compared in terms of area and delay. Table II shows the performance analysis of AES 128-bit implementation.

Table II shows the performance analysis of AES - 128. As previous work, available in literature we have been able to reduce area in terms of LUTs \& Slices to a great extent in 2 stage implementation. The overall delay of 3 stage implementation and 2 stage implementation are very high as their latency is high. In order to reduce delay, we have designed two more architectures called pipelined and combinational. In In terms of overall delay the pipelined architecture is best as cipher text output streams after the 10 clock cycles for encryption and 20 clock cycles for decryption. 
Table II: Performance Analysis of AES - 128

\begin{tabular}{|c|c|c|c|c|c|}
\hline Parameters & $\begin{array}{c}\text { AES - 128 } \\
\text { (3 Stage) } \\
{[\mathbf{1 1}]}\end{array}$ & $\begin{array}{c}\text { AES - 128 } \\
\text { (3 Stage) }\end{array}$ & $\begin{array}{c}\text { AES - 128 } \\
\text { (2 Stage) }\end{array}$ & $\begin{array}{c}\text { AES-128 } \\
\text { (Pipelined) }\end{array}$ & $\begin{array}{c}\text { AES - 128 } \\
\text { (Comb.) }\end{array}$ \\
\hline Number of slices & 7706 & 6302 & 3520 & 26967 & 28820 \\
\hline Slice f/f & $\mathrm{x}$ & 2702 & 2932 & 5324 & $\mathrm{x}$ \\
\hline LUTs & 14884 & 11699 & 6576 & 53536 & 56026 \\
\hline BRAM & $\mathrm{x}$ & $\mathrm{x}$ & 12 & 12 & $\mathrm{x}$ \\
\hline $\begin{array}{c}\text { Latency (encryption/ } \\
\text { decryption) }\end{array}$ & $\mathrm{x}$ & $23 /$ & $39 /$ & $10 / 20$ & $1 / 2$ \\
\hline Delay (ns) & $\mathrm{x}$ & $9.765 \mathrm{~ns}$ & $7.98 \mathrm{~ns}$ & 11.078 & 219.866 \\
\hline $\begin{array}{c}\text { Total Delay (ns) } \\
\text { Latency * Delay }\end{array}$ & $\mathrm{x}$ & $224.595 /$ & $311.22 /$ & $110.78 /$ & $219.866 /$ \\
\end{tabular}

Table III: Performance Analysis of AES - 192

\begin{tabular}{|c|c|c|c|c|}
\hline Parameters & AES - 192 (3 Stage) & AES - 192 (2 Stage) & AES - 192 (Pipelined) & AES - 192 (Comb.) \\
\hline Number of slices & 5523 & 4711 & 33294 & 33573 \\
\hline Slice f/f & 2968 & 4679 & 9322 & $\mathrm{x}$ \\
\hline LUTs & 10576 & 8166 & 62820 & 65214 \\
\hline BRAM & 12 & 12 & 12 & $\mathrm{x}$ \\
\hline Latency (encryption/ & $13 /$ & $47 /$ & $13 /$ & $01 /$ \\
decryption) & 25 & 94 & 21 & 02 \\
\hline Delay (ns) & 12.062 & 12.306 & 10.447 & 236.868 \\
\hline Total Delay (ns) & $156.806 /$ & $578.382 /$ & $135.811 /$ & $236.868 /$ \\
= Latency * Delay & 301.55 & 1156.764 & 219.387 & 473.736 \\
\hline
\end{tabular}

Table III: Performance Analysis of AES - 256

\begin{tabular}{|c|c|c|c|c|}
\hline Parameters & AES - 256 (3 Stage) & AES - 256 (2 Stage) & AES - 256 (Pipelined) & AES - 256 (Comb.) \\
\hline Number of slices & 5333 & 5600 & 39429 & 40153 \\
\hline Slice f/f & 3047 & 5256 & 9517 & $\mathrm{x}$ \\
\hline LUTs & 10160 & 9841 & 76365 & 78148 \\
\hline BRAM & 12 & 10 & 12 & $\mathrm{x}$ \\
\hline Latency (encryption/ & $15 /$ & $55 /$ & $15 /$ & $01 /$ \\
decryption) & 29 & 110 & 23 & 02 \\
\hline Delay (ns) & 12.231 & 13.612 & 12.368 & 283.955 \\
\hline Total Delay (ns) & $183.465 /$ & $748.66 /$ & $185.52 /$ & $283.955 /$ \\
Latency * Delay & 354.699 & 1497.32 & 284.264 & 567.91 \\
\hline
\end{tabular}

Table II shows the performance analysis of AES -192, it can be inferred from table II that, AES 2 stage is the best in terms of area usage as it uses the minimum area, but worst in terms of overall delay. The AES - 192 Pipelined architecture is best in terms of overall delay as it is least and also the output cipher text/plain text streams after 13/21 clock cycles i.e. after each clock new output is generated corresponding to the new input vector. The cost paid is area.

Table III shows the performance analysis of AES - 256, that AES - 2 Stage and AES - 3 stage are almost same in terms of area (resource usage), but AES 2 - Stage is worst in terms of latency and overall delay. So for AES -256 it can be 
interpreted that AES - 2 stage is of not much use as it has highest overall delay and similar area usage comparable to the 3 stage implementation. AES - 256 combinational implementation is best in terms of latency but the overall delay is dependent on combinational delay which is worst in combinational implementation. So, similar to the AES - 128 and AES 192, the pipelined architecture is best in terms of overall delay. The output of encryption and decryption blocks streams after the latency clock cycles (15 and 23 for encryption and decryption respectively).

\section{CONCLUSION}

In this work, we have implemented all the three modules of AES, namely AES - 128, AES 192 and AES - 256. All the three modules are implemented from the four different techniques namely: 3 stage, 2 Stage, Pipelined and combinational. It can be interpreted from the results that 2 stage design is best in low area requirement systems for AES 128 and AES 192, but the area (resource usage) is almost same to 3 stage design in AES - 256 module. The cost paid is overall delay. The pipelined architecture is best in terms of overall delay for the all the three AES modules. Another advantage of using pipelined architecture is that the output of encryption and decryption module streams after specified latency clock cycles i.e. new output is generated after each clock cycle corresponding to the new input. The maximum operating frequency is dependent on delay, and it is the reciprocal of delay (not overall delay), the pipelined architecture has moderate delay in the three modules of AES and hence it can be operated on a high frequency compared to the combinational design.

In this work the three modules of AES works independently and are separate designs, in future three modules can be combined so that a single machine will be able to support all the three modules and also hardware can also be shared to conserve area.

\section{REFERENCES}

[1] Hammad I, El-Sankary K, El-Masry E, "High-speed AES encryptor with efficient merging techniques," IEEE Embedded Systems Letters, 2010, pp.67-71.

[2] I Zhang Y L, Wang X G, "Pipelined implementation of AES encryption based on FPGA," 2010 IEEE International Conference on Information Theory and Information Security, Piscataway: IEEE, 2010, pp. 170173.

[3] Fan C-P, Hwang J-K, "Implementations of high throughput sequential and fully pipelined AES processors on FPGA." ISPACS 2007: Proceeding of 2007 International Symposium on International Signal Processing and Symposium and Communication Systems, Piscataway: IEEE, 2007, pp. 353-356.

[4] Sklavos N, Koufopavlou O, "Architectures and VLSI implementations of the AES-proposal Rijndael," IEEE Transactions on Computers, 2002, 51(12), pp. 14541459.

[5] Borkar A M, Kshirsagar R V, Vyawahare M V, "FPGA implementation of AES algorithm," The 3rd International Conference on Electronics Computer Technology, Piscataway: IEEE, 2011, 3, pp. 401-405.

[6] Joan Daemen,Vincent Rijmen.AES Proposal:Rijdael. The Rijndael Block Cipher.

[7] Vincent Rijmen, "Efficient implementation of the of the rijndael SBox," 2000.

[8] Fischer V, Drutarovsky M, Chodowiec P, "InvMixColunm decomposition and multilevel resource sharing in AES implementations," IEEE Transactions on Very Large Scale Integration Systems, 2005, 13(8), pp. 989-992.

[9] Chien M Ta, Chee Hong Yong, Wooi Gan Yeoh, "A $2.7 \mathrm{~mW}, \quad 0.064 \mathrm{~mm} 2$ linear-in-dB VGA with $60 \mathrm{~dB}$ tuningrange, $100 \mathrm{MHz}$ bandwidth, and two DC offset cancellation loops," IEEE International Workshop on Radio Frequency Integration Technology, Austria: Graz, 2005, pp. 74-77.

[10] J.Balamurugan, Dr.E.Logashanmugam "High Speed Low Cost Implementation of Advanced Encryption Standard on FPGA" ICCET 2014. 